\title{
Non-alcoholic Fatty Liver Disease (NAFLD) and Significant Hepatic Fibrosis Defined by Non-invasive Assessment in Patients with Type 2 Diabetes
}

\author{
Abhasnee Sobhonslidsuk*, Akharawit Pulsombat, Piyaporn Kaewdoung, \\ Supanna Petraksa
}

\begin{abstract}
Background: Non-alcoholic fatty liver disease (NAFLD), the most common liver problem in diabetes, is a risk factor for liver cancer. Diabetes, high body mass index (BMI) and old age can all contribute to NAFLD progression. Transient elastography (TE) is used for non-invasive fibrosis assessment. Objectives: To identify the prevalence of NAFLD and significant hepatic fibrosis in diabetic patients and to assess associated factors. Materials and Methods: One hundred and forty-one diabetic and 60 normal subjects were screened. Fatty liver was diagnosed when increased hepatic echogenicity and vascular blunting were detected by ultrasonography. Liver stiffness measurement (LSM) representing hepatic fibrosis was assessed by TE. LSM $\geq 7 \mathrm{kPa}$ was used to define significant hepatic fibrosis. Results: Four cases were excluded due to positive hepatitis $B$ viral markers and failed TE. Diabetic patients had higher BMI, systolic blood pressure, waist circumference and fasting glucose levels than normal subjects. Fatty liver was diagnosed in $82(60.7 \%)$ diabetic patients but in none of the normal group. BMI (OR: 1.31; 95\% CI: 1.02-1.69; $p=0.038$ ) and alanine aminotransferase (ALT)(OR: 1.14; 95\% CI: 1.05-1.23; $p=0.002$ ) were associated with NAFLD. Diabetic patients with NAFLD had higher LSM than those without [5.99 (2.4) vs $4.76(2.7) \mathrm{kPa}, \mathrm{p}=0.005)]$. Significant hepatic fibrosis was more common in diabetic patients than in normal subjects [22 (16.1\%) vs $1(1.7 \%), p=0.002]$. Aspartate aminotransferase (AST)(OR: 1.24; 95\% CI: 1.07-1.42; $p=0.003$ ) was associated with significant hepatic fibrosis. Conclusions: Sixty and sixteen percent of diabetic patients were found to have NAFLD and significant hepatic fibrosis. High BMI and ALT levels are the predictors of NAFLD, and elevated AST level is associated with significant hepatic fibrosis.
\end{abstract}

Keywords: Diabetes - Non-alcoholic fatty liver disease (NAFLD) - hepatic fibrosis - transient elastography

Asian Pac J Cancer Prev, 16 (5), 1789-1794

\section{Introduction}

Alcohol, hepatitis B and hepatitis C viral infection are common causes of cirrhosis and hepatocellular carcinoma in Thailand and Asian countries (Gao et al., 2012; Somboon et al., 2014). High body mass index (BMI) and type 2 diabetes mellitus are associated with an increased risk of death from cancer and ischemic heart diseases (Fujino, 2007; Chiou et al., 2011). In 2012, more than three hundred and seventy-one million people have diabetes with an increased prevalence of diabetes worldwide. Liver disease is the third most common cause of death in diabetic patients (Tolman et al., 2007). Cryptogenic cirrhosis has become one of the most frequent indications of liver transplantation in the U.S. The spectrum of liver abnormalities in patients with type 2 diabetes includes abnormal liver enzymes, non-alcoholic fatty liver disease (NAFLD), cirrhosis, hepatocellular carcinoma and acute liver failure (Tolman et al., 2007). Mild elevation of serum alanine aminotransferase (ALT) is seen in 2-24\% of diabetic patients (Trombetta et al., 2005). NAFLD is by far the most common cause of a mild elevation of serum ALT in type 2 diabetes (Harris et al., 1998). NAFLD consists of a spectrum of chronic liver diseases from simple steatosis to non-alcoholic steatohepatitis (NASH) and advanced fibrosis (Teli et al., 1995).

Evidence from several longitudinal follow-up studies has confirmed that NASH can progress to advanced fibrosis and cirrhosis (Teli et al., 1995). NAFLD alone or NAFLD coexisting with hepatitis B or C viral infection can progress to cirrhosis (Poortahmasebi et al., 2014). Diabetes, body mass index (BMI), and age are predictors of the presence of advanced fibrosis in NASH (Angulo et al., 2007). Liver biopsy remains the gold standard tool to assess for fibrotic staging of the liver. However, the benefit of liver biopsy comes with the risks of major complications $(1-3 \%)$ and mortality $(0.01 \%)$ in patients undergoing liver biopsy (Bravo et al., 2001). Liver stiffness measurement (LSM) performed by transient elastography (TE) (Fibroscan ${ }^{\circledR}$, Echosen, Paris), which 
uses ultrasound-based technology for quantitative assessment of hepatic fibrosis, has been studied in chronic liver diseases including NAFLD (Wong et al., 2010).

In this study, we aimed to evaluate for the prevalence of NAFLD and significant hepatic fibrosis in patients with diabetes, and to identify the risk factors of NAFLD and significant hepatic fibrosis in these patients.

\section{Materials and Methods}

A cross-sectional study was performed at the liver unit, Ramathibodi Hospital, Mahidol University between October 1st 2011 and September 30th 2012. The study protocol was approved by the Hospital Ethics Committee, and it was carried out according to the Helsinki Declaration guideline. Written informed consent was obtained prior to recruitment. Type 2 diabetic patients and normal subjects were enrolled. Subjects with known history of liver diseases or cirrhosis, regular alcohol drinking more than $20 \mathrm{mg}$ per day and current herbal substance use were excluded. Normal subjects were healthy volunteers with no medical conditions including liver diseases, no history of regular alcohol drinking, normal biochemical tests and negative hepatitis B surface antigen ( $\mathrm{HBsAg}$ ) and antihepatitis $\mathrm{C}$ viral antibody (anti-HCV ab). Demographic data were collected. Body mass index (BMI) was calculated from weight $(\mathrm{kg})$ divided by height ${ }^{2}(\mathrm{~m})$. BMI of 23.0 to $24.9 \mathrm{~kg} / \mathrm{m}^{2}$ for overweight and $\geq 25.0 \mathrm{~kg} / \mathrm{m}^{2}$ for obesity in adult Asians as proposed by the World Health Organization, International Association for the Study of Obesity and International Obesity Task Force in 2000 were employed in this study (WHO, 2000; Alberti et al., 2005). BMI of $25-29.9 \mathrm{~kg} / \mathrm{m}^{2}$ and $\geq 30 \mathrm{~kg} / \mathrm{m}^{2}$ are consistent with obesity class 1 and 2 (WHO, 2000).

Waist circumference was measured at the level of umbilicus in the upright position. Fasting serum samples were taken for hematological and biochemical testing which included complete blood count, liver function test, serum glucose, glycated hemoglobin (HbA1C), lipid panel, hepatitis B and C viral markers.The definition of metabolic syndrome followed the criteria proposed by the International Diabetes Federation (IDF) (Alberti et al., 2005). The IDF criteria require central obesity (: WC $\geq 90 \mathrm{~cm}$ in men and $\geq 80 \mathrm{~cm}$ in women for Asians) plus 2 of the following 4 criteria: triglyceride $\geq 150 \mathrm{mg} / \mathrm{dl}$, HDL cholesterol $<40 \mathrm{mg} / \mathrm{dl}$ in men and $<50 \mathrm{mg} / \mathrm{dl}$ in women, fasting plasma glucose $\geq 100 \mathrm{mg} / \mathrm{dl}$ or presence of type 2 diabetes, and blood pressure $\geq 130 / 85 \mathrm{mmHg}$ or on antihypertensive medication (Alberti et al., 2005).

\section{Abdominal ultrasonography}

Diabetic patients and normal subjects underwent abdominal ultrasonography (HITACHI, Japan) using a convex $3.5 \mathrm{MHz}$ probe for evaluation of fatty liver. The diagnosis of fatty liver was made based on increased liver brightness with presence of sonographic contrasted appearance between liver and kidney parenchyma, high posterior attenuation, reduced vessel wall echogenicity and poorly delineated diaphragm (Hamer et al., 2006).
TE was performed by a single experienced operator using FibroScan (Echosens, Paris, France) with a medium (M) probe operating at a frequency of $3.5 \mathrm{MHz}$. A successful TE examination was defined as $\geq 10$ valid measurements with $>60 \%$ success rate and an interquartile range (IQR) to median ratio of $\leq 0.30$ (Jung and Kim, 2012). The median value of 10 valid measurements was considered to be the liver stiffness measurement (LSM) which was reported as $\mathrm{kPa}$. Significant hepatic fibrosis [or fibrotic (F) stage $\geq 2$ diagnosed by liver biopsy] was defined as LSM $\geq 7 \mathrm{kPa}$ based on the result of a study of LSM using transient elastography in patients with NAFLD (Wong et al., 2010).

\section{Statistical analysis}

Descriptive data were expressed as number (\%) for discrete variables, mean (SD) or median (range) for continuous variables. Statistical analysis of continuous variables was performed by Student's t-test or nonparametric test whatever appropriate. Chi-square test or Fisher exact test was used for analysis of discrete variables. $\mathrm{P}$-value $<0.05$ was considered statistically significant. For variables with P-value $<0.20$ from univariate analysis, they were analyzed further with multivariate analysis using logistic regression or multiple regression analysis as appropriate.

\section{Results}

A total of 141 patients with type 2 diabetes and 60 normal subjects were screened. Four diabetic patients were ineligible for recruitment because of positive $\mathrm{HBsAg}$ in 3 patients and failed TE in 1 patient.

\section{Diabetic patients vs normal subjects}

Demographic data of 137 diabetic patients and 60 normal subjects are shown in Table 1. Diabetic patients were older than normal subjects with mean age (SD) of 60.5 (9.8) vs 39.8 (15.2) years $(\mathrm{p}=0.00)$. Mean BMI and waist circumference were also higher in diabetic patients [26.0 (4.5) vs $23.0(3.3) \mathrm{kg} / \mathrm{m}^{2}$ (p=0.00), and 89.1 (11.4) vs $80.1(9.20) \mathrm{cm}(\mathrm{p}=0.00)]$. Among 137 diabetic patients, $29(21.2 \%), 54(39.4 \%), 22(16.1 \%)$ patients were classified as overweight, obesity class 1 and obesity class 2 according to BMI classification of adult Asians (WHO, 2000). The results of ultrasonography of two diabetic patients were missing. Eighty-two of 135 diabetic patients $(60.7 \%)$ had fatty liver based on ultrasonographic criteria while none in normal group showed evidence of fatty liver from ultrasonography. Mean LSM was significantly higher in diabetic patients than normal subjects $(5.52 \mathrm{vs} 4.16 \mathrm{kPa}$, $\mathrm{p}=0.00)$. Twenty-two patients $(16.1 \%)$ with diabetes had significant hepatic fibrosis, which was defined by LSM $\geq 7.0 \mathrm{kPa}$, comparing to 1 patient $(1.7 \%)$ in normal group $(\mathrm{p}=0.002)$.

\section{NAFLD in diabetic patients}

Out of 135 diabetic patients, ultrasonographic examination revealed fatty liver in 82 patients $(60.7 \%)$ and normal liver in 53 patients $(39.3 \%)$. The prevalence of fatty liver was progressively increased from $17.2 \%$ in normal 
weight patients to $58.6 \%$ in overweight patients, $77.8 \%$ in obesity class 1 and $85.7 \%$ in obesity class 2 diabetic patients $(\mathrm{p}=0.00)$. Seventy-two patients $(60.5 \%)$ out of 119 patients had metabolic syndrome by the IDF definition (Alberti et al., 2005). NAFLD was more commonly seen in diabetic patients with metabolic syndrome than in those without metabolic syndrome [59 (76.6\%) vs 13 (31.0\%), $\mathrm{p}=0.00]$.The characteristics of diabetic patients with and without fatty liver are shown in Table 2. Univariate analysis revealed that diastolic blood pressure (DBP), BMI, WC, aspartate aminotransferase (AST), alanine aminotransferase (ALT), triglyceride (TRIG) levels were significantly higher in diabetic patients with NAFLD than those without NAFLD. Factors with $\mathrm{p}<0.20$ (age, DBP, BMI, WC, AST, ALT, TRIG) were analyzed further with logistic regression analysis. BMI (OR: 1.31; 95\%CI: 1.021.69; $\mathrm{p}=0.038$ ) and ALT (OR: 1.14; 95\%CI: 1.05-1.23; $\mathrm{p}=0.002$ ) were independently associated with the presence of NAFLD in diabetic patients.

\section{Significant hepatic fibrosis in diabetic patients}

LSM, which was used to assess for the severity of hepatic fibrosis, in diabetic patients was significantly higher than in normal subjects [5.52 (2.4) vs 4.16 (0.9)]. TE revealed that twenty-two diabetic patients $(16.1 \%)$ had fibrotic stages of at least significant fibrosis. The characteristics of diabetic patients with or without significant fibrosis are shown in Table 3. Univariate analysis revealed that AST, ALT levels and the diagnosis of fatty liver by ultrasound were significantly related to

Table 1. Demographic Data of Diabetic Patients and Normal Subjects

\begin{tabular}{|c|c|c|c|}
\hline & Normal subjects & Diabetic patients & $\mathrm{p}$-value \\
\hline Number & 60 & 137 & \\
\hline Age* (year) & $39.82(15.2)$ & $60.47(9.8)$ & 0.00 \\
\hline Sex & & & 0.43 \\
\hline Female & $38(63.3 \%)$ & $77(56.2 \%)$ & \\
\hline Male & $22(36.7 \%)$ & $60(43.8 \%)$ & \\
\hline \multicolumn{4}{|l|}{ Blood pressure* $(\mathrm{mmHg})$} \\
\hline Systolic blood pressure & $113.5(15.4)$ & $127.3(15.5)$ & 0.00 \\
\hline Diastolic blood pressure & $70.9(11.0)$ & $73.5(10.7)$ & 0.18 \\
\hline Body mass index $*\left(\mathrm{~kg} / \mathrm{m}^{2}\right)$ & $23.0(3.3)$ & $26.0(4.5)$ & 0.00 \\
\hline Waist circumference* $(\mathrm{cm})$ & $80.1(9.2)$ & $89.1(11.4)$ & 0.00 \\
\hline Aspartate aminotransferase*(u/l) & $24.5(31.1)$ & $25.3(10.9)$ & 0.79 \\
\hline Alanine aminotransferase $*(\mathrm{u} / \mathrm{l})$ & $38.0(30.0)$ & $41.7(19.3)$ & 0.85 \\
\hline Cholesterol*\# (mg/dl) & $200.6(38.1)$ & $185.8(38.5)$ & 0.02 \\
\hline Triglyceride*\# (mg/dl) & $117.0(91.4)$ & $138.0(71.4)$ & 0.16 \\
\hline Fasting glucose $*(\mathrm{mg} / \mathrm{dl})$ & $90.5(7.8)$ & $135.5(41.2)$ & 0.00 \\
\hline Hemoglobin A $1 c^{*}(\%)$ & $5.8(0.5)$ & $7.3(1.4)$ & 0.00 \\
\hline Liver stiffness measurement $*(\mathrm{kPa})$ & $4.2(0.9)$ & $5.5(2.4)$ & 0.00 \\
\hline Interquartile range $(\mathrm{IQR})$ & $0.5(0.4)$ & $0.8(0.9)$ & 0.02 \\
\hline Interquartile range (IQR)/median & $0.1(.01)$ & $0.1(0.1)$ & 0.52 \\
\hline Liver stiffness measurement $\geq 7.0 \mathrm{kPa}$ & $1(1.70 \%)$ & $22(16.1 \%)$ & 0.002 \\
\hline Fatty liver by ultrasonography & $0(0 \%)$ & $82(60.7 \%)$ & 0.00 \\
\hline
\end{tabular}

*mean (SD); \#under treatment

Table 2. Characteristics of Diabetic Patients with and without Non-alcoholic Fatty Liver Disease (NAFLD)

\begin{tabular}{|c|c|c|c|}
\hline & Diabetic patients without NAFLD & Diabetic patients with NAFLD & p-value \\
\hline Number $(\%)$ & $53(39.3)$ & $82(60.7)$ & \\
\hline $\operatorname{Age}^{*}$ (year) & $62.1(11.3)$ & $59.4(8.7)$ & 0.15 \\
\hline Sex & & & 0.32 \\
\hline Female & $28(36.8 \%)$ & $48(63.2 \%)$ & \\
\hline Male & $25(42.4 \%)$ & $34(57.6 \%)$ & \\
\hline \multicolumn{4}{|l|}{ Blood pressure*(mmHg) } \\
\hline Systolic blood pressure & $126.5(17.7)$ & $128.0(14.1)$ & 0.62 \\
\hline Diastolic blood pressure & $70.9(10.7)$ & $75.2(10.5)$ & 0.03 \\
\hline Body mass index $*\left(\mathrm{~kg} / \mathrm{m}^{2}\right)$ & $23.4(3.8)$ & $27.6(4.1)$ & 0.00 \\
\hline Waist circumference* $(\mathrm{cm})$ & $83.0(10.6)$ & $92.9(9.8)$ & 0.00 \\
\hline Aspartate aminotransferase* $(\mathrm{u} / \mathrm{l})$ & $22.4(7.7)$ & $27.3(12.3)$ & 0.007 \\
\hline Alanine aminotransferase $*(\mathrm{u} / \mathrm{l})$ & $32.6(9.0)$ & $47.7(21.9)$ & 0.00 \\
\hline Cholesterol*\# (mg/dl) & $189.8(38.7)$ & $183.9(38.0)$ & 0.40 \\
\hline Triglyceride*\# (mg/dl) & $107.9(52.7)$ & $161.5(75.7)$ & 0.00 \\
\hline Fasting glucose $*(\mathrm{mg} / \mathrm{dl})$ & $132.5(34.9)$ & $137.6(45.3)$ & 0.47 \\
\hline Hemoglobin A1c*(\%) & $7.4(1.5)$ & $7.2(1.3)$ & 0.59 \\
\hline Liver stiffness measurement*(kPa) & $4.8(2.7)$ & $6.0(2.0)$ & 0.005 \\
\hline Interquartile range (IQR) & $0.60(0.3)$ & $0.85(1.1)$ & 0.07 \\
\hline Interquartile range (IQR)/median & $0.13(0.06)$ & $0.14(0.17)$ & 0.61 \\
\hline Liver stiffness measurement $\geq 7.0 \mathrm{kPa}$ & $3(5.7 \%)$ & $18(22.0 \%)$ & 0.008 \\
\hline
\end{tabular}


Table 3. Characteristics of Diabetic Patients with and without Significant Fibrosis by Liver Stiffness Measurement

\begin{tabular}{|c|c|c|c|}
\hline & $\begin{array}{l}\text { Diabetic patients without } \\
\text { significant fibrosis }\end{array}$ & $\begin{array}{l}\text { Diabetic patients with } \\
\text { significant fibrosis }\end{array}$ & p-value \\
\hline Number $(\%)$ & $115(83.9)$ & $22(16.1)$ & \\
\hline Age* (year) $^{*}$ & $59.9(9.9)$ & $63.8(9.2)$ & 0.10 \\
\hline Sex & & & 0.52 \\
\hline Female & $65(84.4 \%)$ & $12(15.6 \%)$ & \\
\hline Male & $50(83.3 \%)$ & $10(16.7 \%)$ & \\
\hline \multicolumn{4}{|l|}{ Blood pressure* $(\mathrm{mmHg})$} \\
\hline Systolic blood pressure & $126.7(15.7)$ & $130.6(14.8)$ & 0.27 \\
\hline Diastolic blood pressure & $73.6(10.5)$ & $73.1(11.9)$ & 0.88 \\
\hline Body mass index $*(\mathrm{~kg} / \mathrm{m} 2)$ & $25.7(4.4)$ & $27.6(5.0)$ & 0.09 \\
\hline Waist circumference* $(\mathrm{cm})$ & $88.1(10.5)$ & $94.2(14.4)$ & 0.07 \\
\hline Aspartate aminotransferase $*(\mathrm{u} / \mathrm{l})$ & $23.1(7.9)$ & $36.5(16.2)$ & 0.001 \\
\hline Alanine aminotransferase*(u/l) & $38.6(14.7)$ & $57.6(30.3)$ & 0.008 \\
\hline Cholesterol*\# (mg/dl) & $186.5(38.3)$ & $181.5(40.9)$ & 0.64 \\
\hline Triglyceride*\# (mg/dl) & $139.8(75.7)$ & $129.3(45.0)$ & 0.42 \\
\hline Fasting glucose* $(\mathrm{mg} / \mathrm{dl})$ & $132.5(35.8)$ & $151.4(60.9)$ & 0.17 \\
\hline Hemoglobin A1c*(\%) & $7.2(1.3)$ & $7.6(1.8)$ & 0.38 \\
\hline Fatty liver by ultrasonography & $64(56.1 \%)$ & $18(85.7 \%)$ & 0.008 \\
\hline
\end{tabular}

*mean (SD); \#under treatment

the presence of significant fibrosis. Factors with $\mathrm{p}<0.20$ (age, BMI, WC, AST, ALT, fasting glucose, presence of NAFLD) were analyzed further with logistic regression analysis. Only AST level was independently associated with significant hepatic fibrosis (OR: 1.24; 95\%CI: 1.07 $1.42 ; \mathrm{p}=0.003)$. Eight patients $(5.93 \%)$ had $\mathrm{LSM} \geq 8.7 \mathrm{kPa}$ which was suggested to be the LSM cutoff of advanced fibrosis in NAFLD patients (Wong et al., 2010). NAFLD was detected in 6 patients $(75 \%)$ who had LSM $\geq 8.7 \mathrm{kPa}$. One patient had LSM of $21.8 \mathrm{kPa}$. Liver biopsy was done in this case and cirrhosis was confirmed. NAFLD was likely to be the cause of advanced fibrosis or cirrhosis in these 8 patients, except for 1 case who had history of regular alcohol drinking for 30 years and quit alcohol drinking five years ago.

\section{Factors related to liver stiffness measurement (LSM)}

From univariate analysis of the diabetic patient data, it revealed that BMI, WC, AST, ALT and presence of fatty liver by ultrasonography were significantly related to LSM. From multiple regression analysis, AST was independently associated with LSM (coefficient of 0.1 , standard error of 0.03 and p-value of 0.0005$)$.

\section{Discussion}

The clinical entity of NAFLD ranges from benign simple steatosis to non-alcoholic steatohepatitis (NASH). Hepatic fibrosis is frequently associated with NASH, and about $10 \%$ of NASH patients can progress to cirrhosis (Teli et al., 1995). The risk of hepatocellular carcinoma increases after the development of liver cirrhosis (Teli et al., 1995). Patients with diabetes, similar to those with dyslipidemia, hypertension, or other metabolic syndrome, are at risk of NAFLD (Cusi, 2009). Hyperinsulinemia and hyperglycemia in diabetes promote lipogenesis by upregulating hepatic sterol regulatory element binding protein 1c (SREBP1c) and carbohydrate regulatory element binding protein (ChREBP) activity (Adiels et al., 2008; Cusi, 2009). Insulin-resistant adipose tissue in diabetes is another factor that promotes the development of steatosis (Adiels et al., 2008; Cusi, 2009). In addition, de novo lipogenesis stimulated by high carbohydrate and fructose intake lead to the activation of hepatic inflammatory pathway (Adiels et al., 2008; Cusi, 2009). Previous studies have confirmed that NAFLD patients with diabetes are at a higher risk for the progression to advanced fibrosis and cirrhosis than those without diabetes (Teli et al., 1995; Younossi et al., 2004).

Diabetes is seen in $18-33 \%$ of patients with NAFLD. On the contrary, $49-69.4 \%$ of patients with diabetes are reportedly to have NAFLD (Younossi et al., 2004; Trombetta et al., 2005; Tolman et al., 2007; Cusi, 2009; Leite et al., 2009). NAFLD is the most common cause of serum ALT elevation in diabetes (Younossi et al., 2004). In this study, the prevalence of NAFLD in diabetic patients at out-patient clinics of our hospital, which is a tertiary care hospital, was about $60 \%$. The reported prevalence of NAFLD in diabetes varies according to the methods used to evaluate the liver. The gold standard for steatosis and fibrosis assessment is liver biopsy (Schwenzer et al., 2009; Hernaez et al., 2011). However, liver biopsy is an invasive technique which can yield minimal risks of morbidity and mortality. MR spectroscopy (MRS) is a non-invasive, highly accurate technique for fat quantification, because it measures proton signal from hepatocyte triglyceride stores directly (Schwenzer et al., 2009). However, a high cost of MRS technique, which is not universal accessibility and a long period of time required for examination are important obstacles of MRS study. Ultrasonography is inexpensive and widely available. It has 60-94\% sensitivity and 66-97\% specificity for grading of hepatic steatosis (Schwenzer et al., 2009; Hernaez et al., 2011). However, ultrasonography detects fat in the liver only when there is hepatic fat accumulation more than $33 \%$ (Schwenzer et al., 2009; Hernaez et al., 2011). We showed that the predictive factors of having NAFLD identified by ultrasonography in diabetes patients were BMI (OR: 1.31; 95\%CI: 1.02-1.69; $\mathrm{p}=0.038$ ) and ALT level (OR: 1.14; 95\%CI: $1.05-1.23 ; \mathrm{p}=0.002)$. The prevalence of 
NAFLD was more commonly seen in diabetic patients with obesity class 1 and 2, and in diabetic patients with fulfilled criteria of metabolic syndrome. The result of this study is consistent with some conclusion from a previous report which showed the association of abdominal obesity, high ALT level and hypertriglyceridemia with the presence of NAFLD in diabetes patients (Leite et al., 2009).

Though liver biopsy is a gold standard for fibrosis assessment in chronic liver disease and NAFLD, it cannot be done routinely due to the risks of complications and death. Noninvasive fibrosis assessment includes liver enzymes, serum biomarkers and transient elastography (TE) which has been studied in chronic liver diseases including NAFLD. Serum biomarkers such as CK18 levels and CK18 have been developed to replace liver biopsy. CK18, which is a marker of necrosis has been used for differentiating NASH from simple steatosis (Jacqueminet et al., 2008). From a study of CK18 in diabetic patients, $4.3 \%$ of patients were found to have advanced fibrosis (Jacqueminet et al., 2008). The results of LSM study in various chronic liver diseases using TE have been reported. TE is more accurate than biologic markers for fibrosis assessment in NAFLD (Wong et al., 2010). The best cutoffs of LSM in NAFLD for F2 staging or greater, F3 staging or greater and F4 are 7.0, 8.7 and $10.3 \mathrm{kPa}$, respectively (Wong et al., 2010). By using the LSM cutoffs from the previous study (Wong et al., 2010), we found that 22 diabetic patients $(16.1 \%)$ had significant hepatic fibrosis (LSM $\geq 7 \mathrm{kPa}$ ) and 8 patients $(5.84 \%)$ had advanced fibrosis or higher (LSM $\geq 8.7 \mathrm{kPa})$. In this study, the prevalence of diabetic patients with significant fibrosis as assessed by TE was lower than the result from a recent study of diabetic patients in Australia (Casey et al., 2012). In that report, $26 / 74$ patients (35\%) had LSM $\geq 7.65 \mathrm{kPa}$, with biopsy-confirmed significant fibrosis in $12 / 16$ patients and cirrhosis in 2 patients (Casey et al., 2012). The difference in prevalence of significant fibrosis in the diabetic Australian study (Casey et al., 2012) and ours may be explained by the higher BMI and WC of the Australian patients when compared to our patients [(32.8 vs $\left.25.97 \mathrm{~kg} / \mathrm{m}^{2}\right)$ and $(111.3$ vs $\left.89.08 \mathrm{~cm})\right]$. Likewise, higher mean LSM and prevalence of NAFLD were seen in Australian patients than in our Thai patients [(8.3 vs $5.52 \mathrm{kPa}$ ) and (70 vs.60.7\%)]. Although AST, ALT level and NAFLD were found to be significant factors in univariate analysis, similar to the results from the previous study (Casey et al., 2012), AST level alone was associated with significant fibrosis (OR: 1.24 ; 95\%CI: $1.07-1.42 ; \mathrm{p}=0.003)$. Furthermore, AST level was found to be an independent factor relating to LSM from multiple regression analysis.

Lack of liver biopsy in every case to confirm hepatic fibrotic staging in this study is the crucial limitation of our report. However, we avoided the inaccuracy of TE measurement by following the standard protocol for TE performance issued by Echosen company, and the TE machine has had regularly inspected and calibrated. About 60 percent and 16 percent of diabetic patients had NAFLD and significant hepatic fibrosis by noninvasive assessment. High BMI and elevated ALT level were the predictors of NAFLD in diabetic patients. The predictive factor of significant fibrosis in diabetic patients was AST level. However, further studies are required to confirm the results of this study.

\section{Acknowledgements}

The study was funded by the Gastroenterological Association of Thailand. The authors would like to sincerely thank Professor Amnuay Thithapandha for his critical and invaluable comments.

\section{References}

Adiels M, Taskinen MR, Boren J (2008). Fatty liver, insulin resistance, and dyslipidemia. Curr Diabetes Rep, 8, 60-4.

Alberti KGM, Zimmet P, Shaw J (2005). The metabolic syndrome: a new worldwide definition. Lancet, 366, 105962.

Angulo P, Hui JM, Marchesini E, et al (2007). The NAFLD fibrosis score: a noninvasive system that identifies liver fibrosis in patients with NAFLD. Hepatology, 45, 846-54.

Bravo AA, Sheth SG, Chopra S (2001). Liver biopsy. $N$ Engl $J$ Med, 344, 495-500.

Casey SP, Kemp WW, Mclean CA, et al (2012). A prospective evaluation of the role of transient elastography for the detection of hepatitis fibrosis in type 2 diabetes without liver disease. Scand J Gastroenterol, 47, 836-41.

Chiou WK, Huang BY, Chou WY, et al (2011). Incidences of cancers in diabetic and non-diabetic hospitalized adult patients in Taiwan. Asian Pac J Cancer Prev, 12, 1577-81.

Cusi K (2009). Nonalcoholic fatty liver disease in type 2 diabetes mellitus. Curr Opin Endocrinol Diabetes Obes, 16, 141-9.

Fujino Y (2007). Anthropometry, development history and mortality in the Japan collaborative cohort study for evaluation of cancer (JACC). Asian Pac J Cancer Prev, 8, 105-12.

Gao J, Xie L, Yang WS, et al (2012). Risk factors of hepatocellular carcinoma--current status and perspectives. Asian Pac J Cancer Prev, 13, 743-52.

Hamer OW, Aguirre DA, Casola G, et al (2006). Fatty liver: Imaging patterns and pitfalls. Radiographics, 26, 1637-53.

Harris MI, Flegal KM, Cowie CC, et al (1998). Prevalence of diabetes, impaired fasting glucose, and impaired glucose tolerance in U.S. adults: the Third National Health and Nutrition Examination Survey, 1988-1994. Diabetes Care, 21, 518-24.

Hernaez R, Lazo M, Bonekamp S, et al (2011). Diagnostic accuracy and reliability of ultrasonography for the detection of fatty liver: A meta-analysis. Hepatology, 54, 1082-90.

Jacqueminet S, Lebray P, Morra R, et al (2008). Screening for liver fibrosis by using a noninvasive biomarker in patients with diabetes. Clin Gastroenterol Hepatol, 6, 828-31.

Jung KS, Kim SU (2012). Clinical applications of transient elastography. Clin Mol Hepatol, 18, 163-73.

Leite NC, Salles GF, Araujo ALE, et al (2009). Prevalence and associated factors of non-alcoholic fatty liver disease in patients with type2 diabetes mellitus. Liver Int, 29, 113-9.

Poortahmasebi V, Alavian SM, Keyvani H, et al (2014). Hepatic steatosis: prevalence and host/viral risk factors in Iranian patients with chronic hepatitis B infection. Asian Pac J Cancer Prev, 15, 3879-84.

Schwenzer NF, Springer F, Schraml C, et al (2009). Noninvasive assessment and quantification of liver steatosis by ultrasound, computed tomography and magnetic resonance. J Hepatol, 51, 433-55.

Somboon K, Siramolpiwat S, Vilaichone RK (2014). 
Abhasnee Sobhonslidsuk et al

Epidemiology and survival of hepatocellular carcinoma in the central region of Thailand. Asian Pac J Cancer Prev, 15, 3567-70.

Teli MR, James OF, Burt AD, et al (1995). The natural history of nonalcoholic fatty liver: a follow-up study. Hepatology, 22, 1714-9.

Tolman KG, Foneca V, Dalpiaz A, et al (2007). Spectrum of liver disease in type 2 diabetes and management of patients with diabetes and liver disease. Diabetes Care, 30, 734-43.

Trombetta M, Spiazzi G,Zoppini G, et al (2005). Review article: type 2 diabetes and chronic liver disease in the Verona diabetes study. Alimen Pharmacol Ther, 22, 24-7.

WHO (2000). World health organisation/international association for the study of obesity/international obesity taskforce. the asia-pacific perspective: redefining obesity and its treatment. http://www.idf.org.au/obesity report.htm.

Wong VWS, Vergniol J, Wong GLH, et al (2010). Diagnosis of fibrosis and cirrhosis using liver stiffness measurement in nonalcoholic fatty liver disease. Hepatology, 51, 454-62.

Younossi ZM, Gramlich T, Matteoni CA, et al (2004). Nonalcoholic fatty liver disease in patients with type 2 diabetes. Clin Gastroenterol Hepatol, 2, 262-5. 